Cinémas

Revue d'études cinématographiques

Journal of Film Studies

\title{
Le panorama et les origines de la reconstitution cinématographique
}

\section{Alison Griffiths}

Volume 14, numéro 1, automne 2003

Dispositif(s) du cinéma (des premiers temps)

URI : https://id.erudit.org/iderudit/008957ar

DOI : https://doi.org/10.7202/008957ar

Aller au sommaire du numéro

Éditeur(s)

Cinémas

ISSN

1181-6945 (imprimé)

1705-6500 (numérique)

Découvrir la revue

Citer cet article

Griffiths, A. (2003). Le panorama et les origines de la reconstitution cinématographique. Cinémas, 14(1), 35-65. https://doi.org/10.7202/008957ar
Résumé de l'article

Cet article se penche sur quelques-unes des propriétés du panorama qui, sur les plans phénoménologique et esthétique - mais aussi idéologique augurent certains traits fondamentaux du cinéma. La reconstitution, en tant que principe structurant qui préside à « l'effet panorama ", permet d'établir certaines correspondances entre les deux médias. Les panoramas dépeignant les grandes batailles historiques s'avèrent, à cet égard, particulièrement utiles pour dégager un certain nombre de procédés d'organisation spatio-temporelle qui anticipent les procédés qu'on trouvera bientôt dans les vues animées. 


\section{Le panorama et les origines de la reconstitution cinématographique}

\section{Alison Griffiths}

\section{RÉSUMÉ}

Cet article se penche sur quelques-unes des propriétés du panorama qui, sur les plans phénoménologique et esthétique - mais aussi idéologique —, augurent certains traits fondamentaux du cinéma. La reconstitution, en tant que principe structurant qui préside à "l'effet panorama», permet d'établir certaines correspondances entre les deux médias. Les panoramas dépeignant les grandes batailles historiques s'avèrent, à cet égard, particulièrement utiles pour dégager un certain nombre de procédés d'organisation spatiotemporelle qui anticipent les procédés qu'on trouvera bientôt dans les vues animées.

\section{ABSTRACT}

This article considers some of the characteristics of the panorama which, on phenomenological, aestheticbut also ideological—levels, intimate some fundamental traits of film. Certain correspondences between the two media may be surmised in the operation of reconstitution, insofar as it is a structural principle that directs the overall "panoramic effect." In this respect, the panoramas depicting great historical battles prove to be particularly useful in pointing out a number of methods of spatial-temporal organisation which anticipate those to be found later in moving pictures.

\section{«La sensation circulaire» : cinéma et premiers panoramas}

Un postulat accepté depuis longtemps par l'ensemble des historiens du cinéma veut que les panoramas du XIX siècle aient 
été d'importants précurseurs des vues animées. En effet, bien avant que les historiens contemporains de l'art et autres théoriciens de la culture ne relient l'hyperréalisme de ces gigantesques peintures immersives au mimétisme mécanisé de l'image cinématographique, de nombreux historiens des générations précédentes avaient tenté le rapprochement entre les deux modes de représentation. En 1933, Monas N. Squires (1933, p. 246) avançait que les panoramas des décennies 1840 et 1850 constituaient de véritables "ancêtres du cinéma moderne», une idée reprise trois ans plus tard par Bertha L. Heilbron (1936, p. 132133), qui employait pour sa part l'expression "films de voyage " pour faire référence aux panoramas du XIX siècle. En 1959, le spécialiste du panorama, Joseph E. Arrington (1959, p. 14), soutenait dans le même esprit que le panorama avait sans aucun doute été "l'ancêtre préphotographique du cinéma", tandis qu'en 1965, dans son ouvrage Archéologie du cinéma, C.W. Ceram se penchait sur plusieurs panoramas qui auraient agi comme précurseurs des vues animées.

Si l'analogie entre le cinéma et les panoramas semble, à plusieurs égards, s'imposer d'elle-même, les raisons profondes qui la sous-tendent ne sont pourtant pas facilement identifiables. En effet, la tâche se complique lorsque vient le temps de déterminer comment les formes représentationnelles du panorama anticipent le cinéma ou d'éclaircir en quoi exactement les normes opérationnelles et esthétiques du panorama constituent de véritables signes précurseurs du média cinématographique. En plus de recenser, entre les deux médias, certaines correspondances générales d'ordre textuel ou phénoménologique (par exemple, comment l'échelle et l'hyperréalisme conféraient au panorama un caractère protocinématographique), le présent article propose une analyse discursive mettant en lumière la façon dont le panorama était appréhendé en termes distinctement cinématographiques par certains spectateurs. Afin d'éclaircir la nature du lien unissant ces deux modes de représentation, nous nous attarderons plus particulièrement sur la reconstitution, qui agit comme principe structurant autant dans le cinéma des premiers temps que dans le panorama. En plus d'agir comme principe structurant qui préside à l'élaboration de plusieurs panoramas 
non fictionnels ${ }^{1}$, la reconstitution permet de définir l'idée même de l'"effet panorama». En effet, les panoramas misent essentiellement sur le réinvestissement, sur l'appréhension renouvelée - sous une forme différente - d'un événement survenu dans un temps et un lieu différents de ceux, précisément, de la reconstitution. Prétendant à une reconstruction authentique et topographiquement exacte de certains combats, paysages ou autres vestiges antiques (telle l'Acropole d'Athènes), les panoramas s'appropriaient la réalité historique et géographique sur la base d'un rapport indiciel, tout comme ce sera le cas quelques années plus tard pour le cinéma. Toutefois, si le panorama anticipait aux plans phénoménologique et discursif quelques-unes des propriétés de la reconstitution cinématographique, certaines différences fondamentales demeurent. En effet, si le panorama est en mesure de reconstruire un paysage ou une scène tirée de l'histoire ou des grands titres des journaux, il lui est en revanche impossible - puisqu'il propose une image figée dans le temps — de reconstituer intégralement l'événement pour le spectateur. En d'autres mots, la scène reconstruite par le panorama n'est pas littéralement rejouée pour le spectateur, comme elle pourra l'être dans le cadre de la reconstitution cinématographique d'une bataille ou d'une exécution. La peinture ne contient, au sens strict du terme, aucune action. Les panoramas ne pouvaient que suggérer le mouvement: par le déplacement physique de la toile devant les spectateurs dans le cas des panoramas défilants (figure 1) ou par des effets de lumière suggérant le passage du temps dans le cas du diorama de Daguerre. Si les possibilités offertes par les reconstitutions intégrales du cinéma demeuraient hors de la portée des exploitants du panorama, ces derniers savaient néanmoins pallier cette limitation en insistant de manière explicite sur les propriétés reconstitutives du panorama. Le spectateur était ainsi encouragé à appréhender les événements représentés devant lui comme s'il s'agissait d'actions se déroulant dans un espace et un temps à la fois continus et immédiats. La reconstitution considérée comme trope cinématographique pourrait ainsi fournir un cadre théorique susceptible de mener à une meilleure compréhension des liens formels unissant panorama et cinéma des premiers temps. 
CiNéMAS, vol. 14, n 1

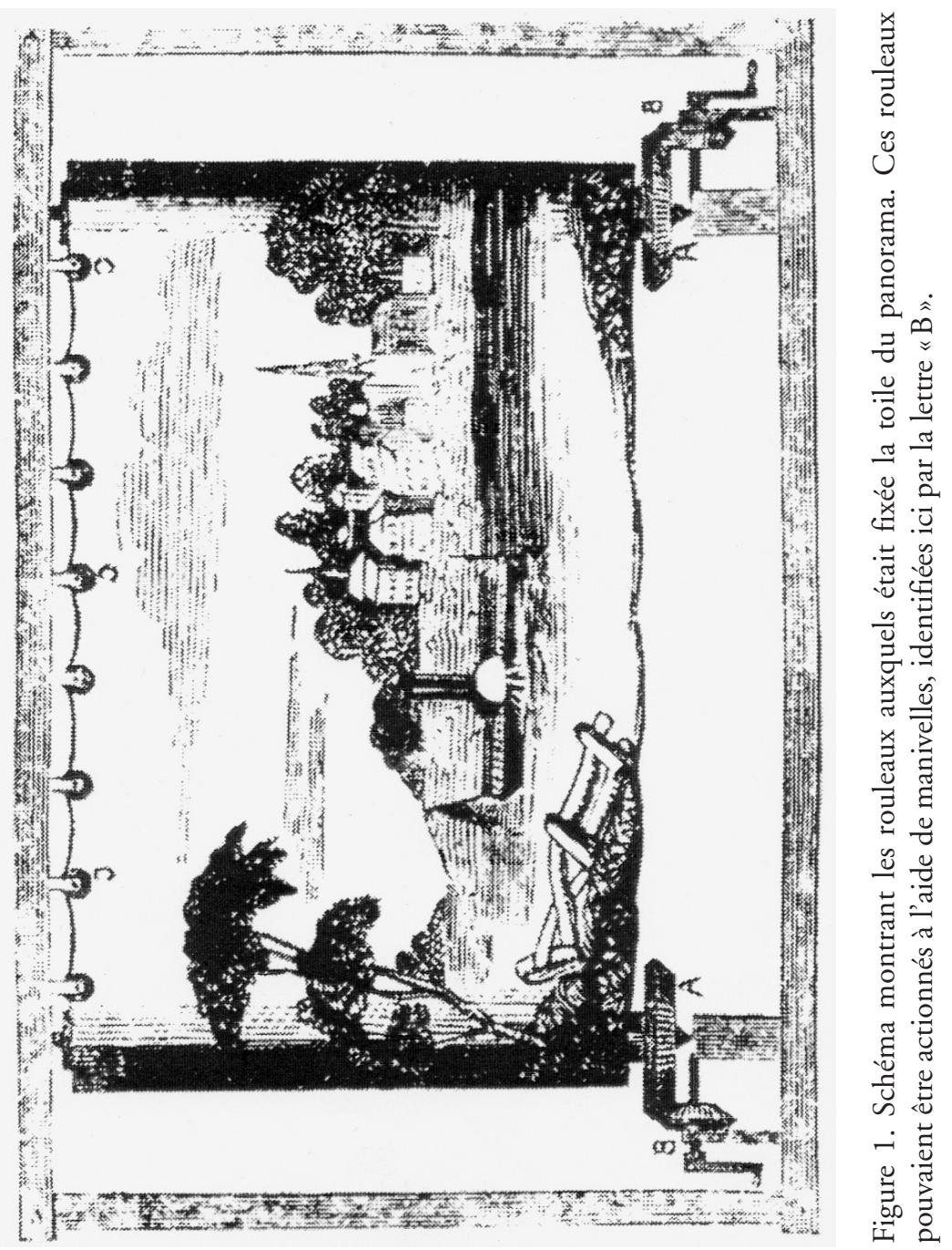


Certaines idées reçues dans le champ des études cinématographiques ont assigné à la reconstitution un rôle pour le moins ambigu. Si la reconstitution a généralement été identifiée comme un procédé récurrent, tant dans les premières actualités filmées que dans les docudrames contemporains, elle semble avoir généré beaucoup moins d'explications théoriques que sa présence massive dans la production hollywoodienne et dans l'«infospectacle» télévisuel aurait pu en motiver. Ainsi, même si la littérature "classique" sur le documentaire a souvent abordé de manière descriptive et normative le phénomène de la reconstitution, on a encore accordé que très peu d'attention à la manière dont elle préfigure, dans les formes précinématographiques de divertissement, le rapport que le cinéma documentaire établira avec son spectateur.

À cheval entre l'attraction populaire et le divertissement mondain, les panoramas agissent en quelque sorte comme des textes interstitiels, qui amalgament discours promotionnels et techniques artistiques diverses, empruntées aussi bien aux beaux-arts qu'aux amusements populaires. Cette caractéristique faisait du panorama un média particulièrement propice aux reconstitutions. En présentant des fac-similés d'événements et de lieux géographiques véritables, les promoteurs du XIX ${ }^{\mathrm{e}}$ siècle exploitaient le mode d'adresse spectaculaire caractéristique du panorama. En effet, une visite au panorama promettait une expérience unique: celle d'une représentation immersive de lieux et d'événements historiques véritables, peinte de façon à accentuer la fidélité et la vraisemblance de l'ensemble. Bien que la peinture de chevalet réaliste ait eu, à cette époque, des prétentions similaires en ce qui a trait à la vraisemblance, trois facteurs n'en placent pas moins le panorama dans une situation unique à titre de précurseur du cinéma. Premièrement, le mode de réception sollicité par l'échelle du panorama: contrairement aux peintures ou aux photographies, les immenses toiles du panorama remplissaient entièrement le champ de vision du spectateur. Deuxièmement, le statut particulier du panorama en tant que technologie permettant un voyage virtuel, capable d'évoquer le réel, était un élément constitutif de l'expérience panoramique. Le troisième facteur est plus spécifique au panorama 
défilant: il s'agit du contexte de présentation, qui exigeait du spectateur l'adoption d'une position fixe. Celui-ci ne déambule pas devant la toile, mais s'assoit plutôt devant elle afin de la regarder se dérouler lentement dans la pénombre de l'auditorium, pour la durée d'une représentation qui est agrémentée d'un accompagnement musical et du commentaire d'un conférencier (figure 2). Toutefois, avant d'examiner la relation complexe qui unit les panoramas du XIX siècle et les pratiques développées par le cinéma des premiers temps, un bref survol des origines du panorama s'impose.

Malgré les fluctuations de popularité dont il a été l'objet tout au long du XIX ${ }^{e}$ siècle, le panorama compte parmi les premières (et les plus rentables) formes de divertissement visuel populaire. Breveté par l'Irlandais Robert Barker en $1787^{2}$, le premier panorama, intitulé $A$ View of London, fut présenté en primeur au Leicester Square de Londres en janvier 1792. Il connaît un tel succès que Barker s'empresse d'agrandir la toile originale, qui formait un demi-cercle, en un cercle entier. Suite au succès phénoménal de ce premier panorama, Barker construit en 1794, sur Leicester Square, une rotonde d'une trentaine de mètres, appelée "The Panorama», qui renferme également une promenade circulaire surélevée destinée à la mise en scène de peintures panoramiques de taille plus modeste (figure $3^{3}$ ). Le velum (un auvent en forme d'ombrelle couvrant l'espace prévu pour les spectateurs) ainsi que la partie inférieure de la peinture panoramique sont masqués par un morceau d'étoffe d'une même couleur, tendu entre la plate-forme et la toile ${ }^{4}$. Investi d'une nouvelle omniscience, le spectateur se trouvait ainsi immergé dans une réalité artificielle au sein de laquelle toute frontière séparant le réel de l'artifice avait, peu ou prou, été éliminée. Privé de toute forme de repère spatial pouvant subordonner la peinture à un espace second, qui l'engloberait, le spectateur se trouvait plus disposé à accepter l'illusion que si la peinture avait été encadrée, ou délimitée, de façon conventionnelle (figure 4). L'espace "cadré" de la peinture agit comme une fenêtre s'ouvrant sur un espace "illusoire», tandis que l'espace "sans limite» du panorama vise à produire chez le spectateur l'impression qu'il est transporté, littéralement, au cœur de l'espace représenté. 


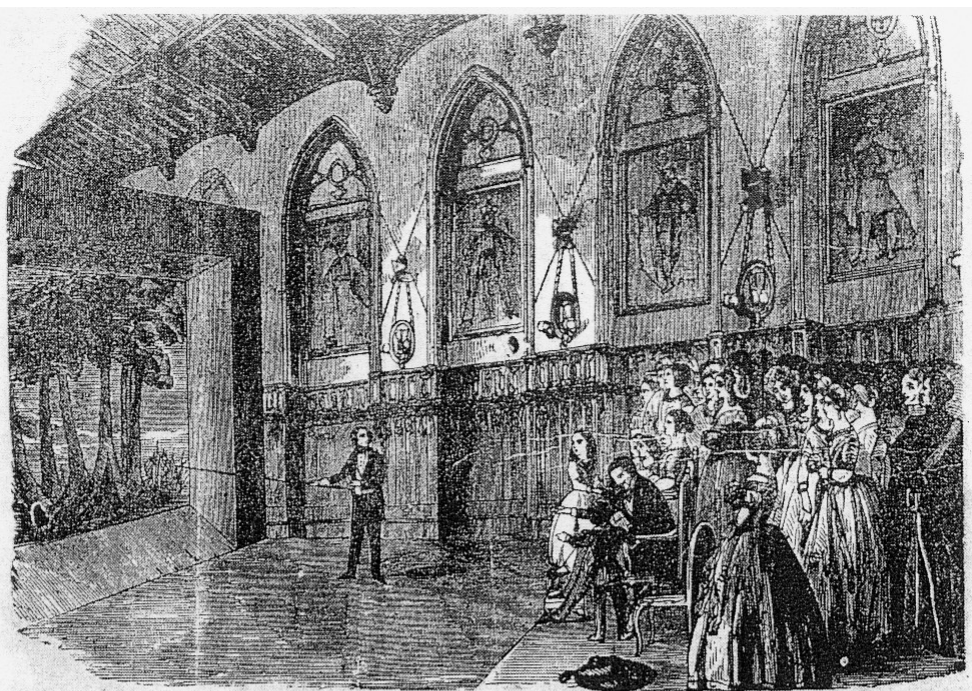

Mr. BANVARD exhibiting and explaining his MAMMOTH PANORA MA OF THE MISSISSIPPI, before the Queen, Prince Aibert, the Royal Children, and the Court, at St.George's Hall, Windsor Castle. At the Close of the Exhilition, HER MAJESTY Wa

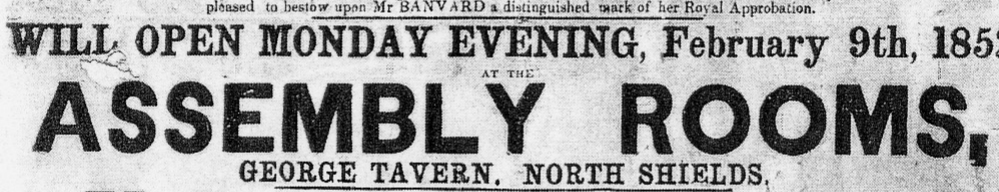

1.

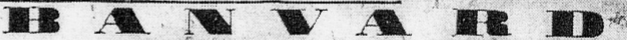
Has the horior of anuouncing that his GIGANTIC, ORIGINAL, MOVING PANORAMA of the

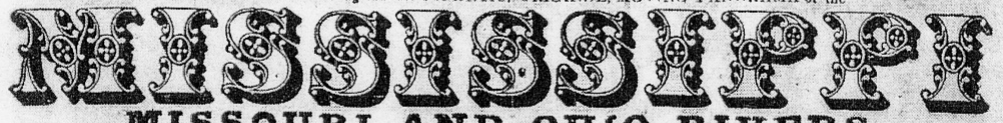
MISSOURI AND OFIO RIVERS,

QUNANANIDCOURT, AT WINDSOR CASTLE,

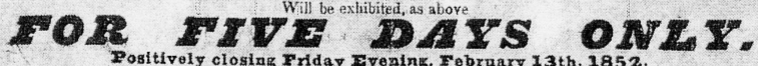

Fabibition oa flonday, once only : in the Evening at half past 8 o'clock. On Tresday and Wednesalay, And on Thurst ay and Free

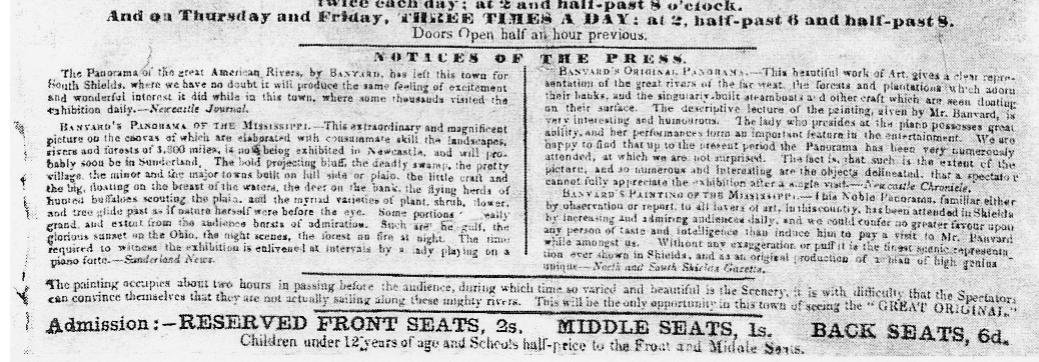

Figure 2. Banvard présentant son panorama du Mississippi à la reine Victoria, en 1852. 


\section{CiNéMAS, vol. 14, n 1}

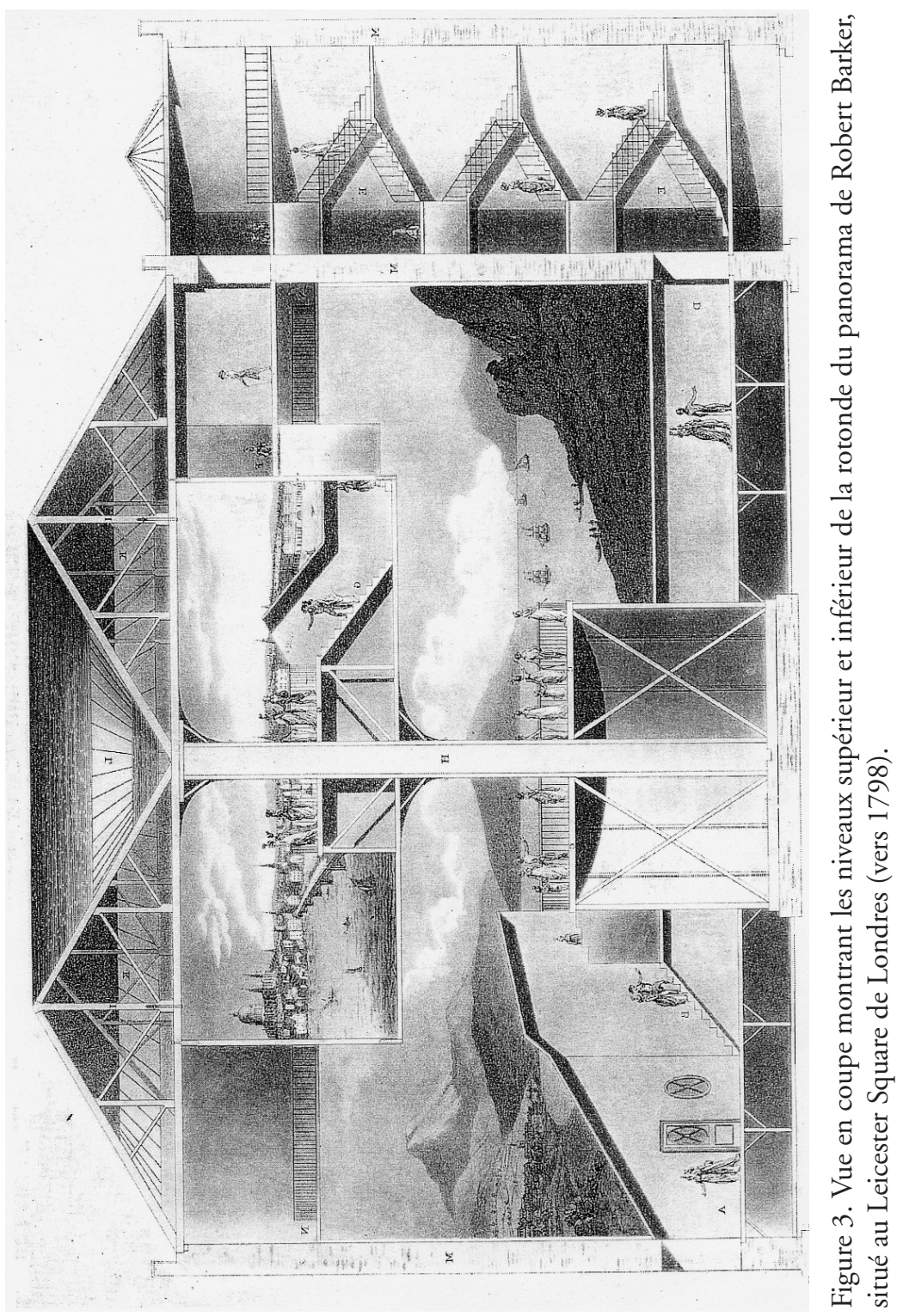


Le panorama et les origines de la reconstitution cinématographique

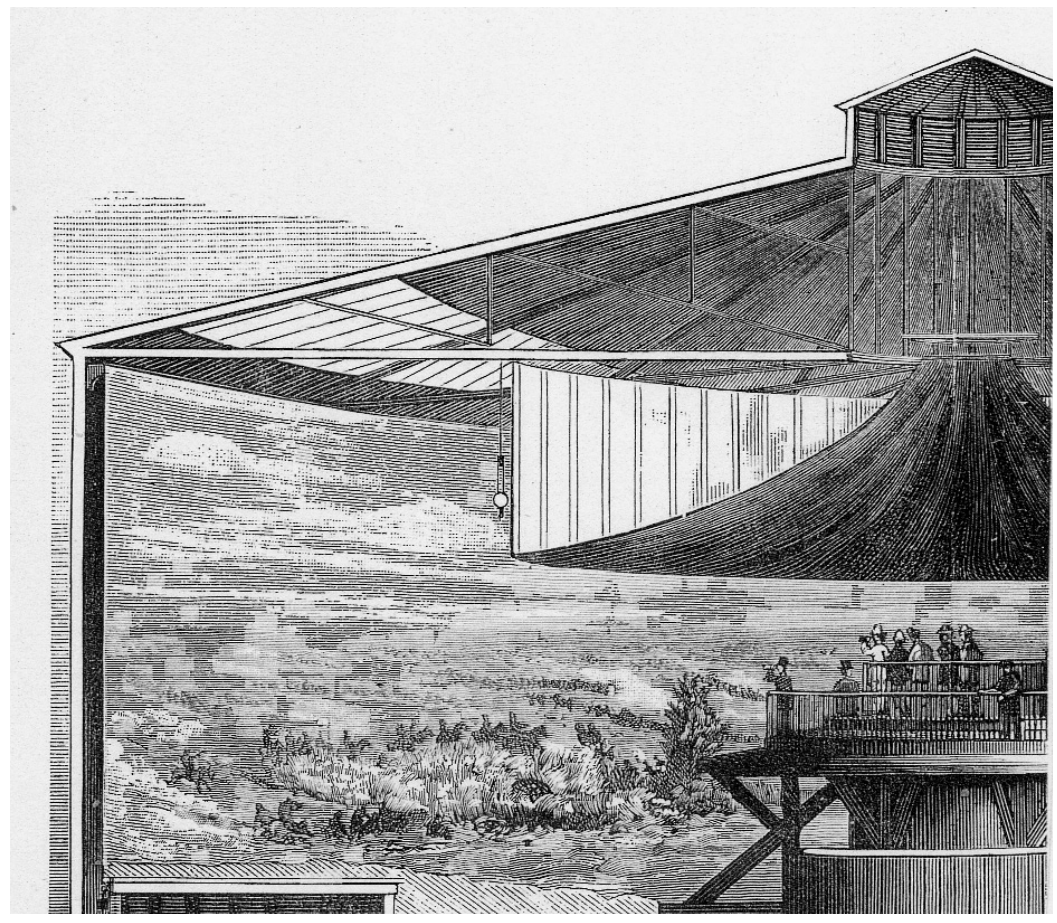

Figure 4. Spectateurs sur la plate-forme d'observation du panorama The Battle of Gettysburg, de Paul Philippoteaux (1884). 
D'autre part, comme le laisse supposer la figure 5, qui montre un groupe de visiteurs souffrant du mal de mer sur la plateforme d'un panorama, le mode de réception induit par le panorama pouvait générer une sociabilité (et une nausée...) d'un ordre différent de celui que suscitait la pénombre des salles du nickelodeon.

Les méthodes utilisées en vue de recréer les événements évoluèrent progressivement, allant même jusqu'à incorporer la temporalité comme principe structurant. Les premiers panoramas privilégiaient une représentation spatiale et temporelle unitaire, montrant par exemple ce que les spectateurs auraient pu contempler, en un coup d'œil, depuis le sommet d'un édifice ou d'une montagne: c'est ce que je désigne sous le vocable de "panorama naturaliste». On en vint ensuite, notamment dans les panoramas représentant des scènes d'action, à construire la scène comme une vue "composite", qui rassemblait divers incidents disparates ayant eu lieu à l'occasion d'une grande bataille, ou de tout autre grand événement. Ces incidents discontinus étaient ainsi intégrés dans un champ visuel uniforme, d'apparence continue, qui s'étalait devant l'observateur sur 360 degrés.

Il y a donc une importante distinction à faire entre l'illusionnisme presque parfait du "panorama naturaliste», non composite, qui reste unitaire sur les 360 degrés du champ visuel, et l'artifice relatif du "panorama composite". Même si ces approches peuvent toutes deux être reliées au cinéma, elles n'anticipent pas moins le «cinématographique» de façon nettement différente. Si, dans sa préfiguration de l'illusion spatiale du cinéma, le panorama naturaliste demeura fidèle à la conception initiale de Robert Barker d'une "vision panoramique " circulaire, le panorama composite annonçait plutôt les perspectives multiples, la narration et la sélection du détail des premiers films pluriponctuels.

On distribuait généralement au spectateur des deux types de panoramas des guides illustrés, permettant d'orienter son regard sur les gigantesques toiles. Il s'agissait de plans sur lesquels les centres d'intérêt offerts au regard étaient répertoriés et identifiés par un numéro (figure 6). Mesurant approximativement $40 \mathrm{x}$ 
le PANORAMA DU "VENGEUR,

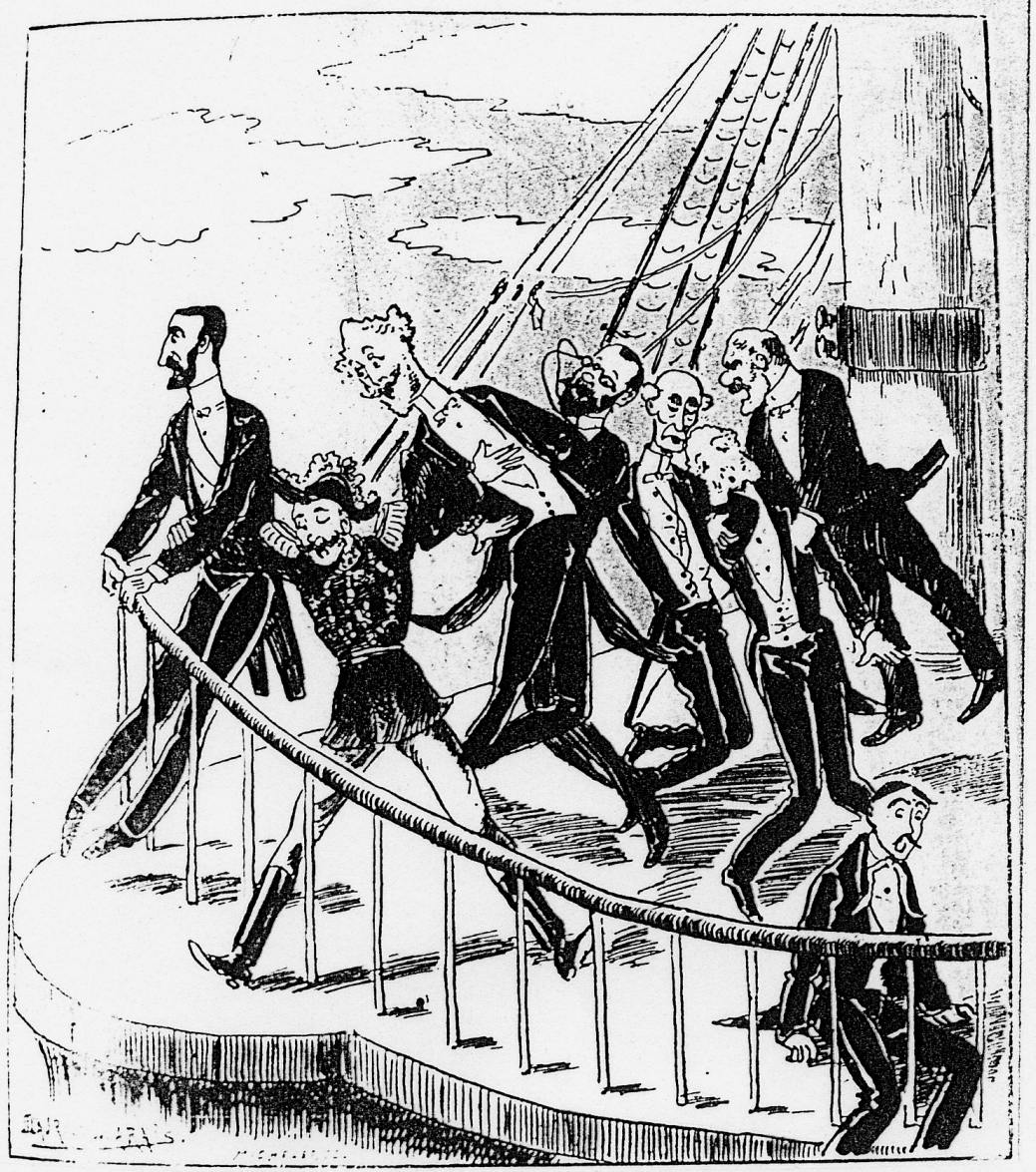

Figure 5. Dessin humoristique intitulé Le Panorama du "vengeur" (vers 1880-1890). 
$20 \mathrm{~cm}$, le plan était habituellement intégré au livret, vendu sur le site du spectacle. Il était normalement inséré dans la pochette de couverture et le spectateur pouvait l'en sortir pour le consulter et soumettre à un examen plus approfondi les centres d'intérêt qui y étaient énumérés, le premier de ceux-ci étant invariablement localisé dans le coin supérieur gauche (les panoramas étaient toujours divisés en deux fragments de 180 degrés chacun, figurant l'un au-dessus de l'autre). En scrutant le plan de gauche à droite, le spectateur pouvait approfondir ses connaissances en se référant à la section du livret fournissant des informations plus substantielles sur la plupart - sinon la totalité - des points numérotés ${ }^{5}$. En organisant les "vues» comme une succession d'attractions qui se présentaient selon un ordre déterminé à l'avance, ces plans codifiaient et ordonnaient le regard prétendument autonome du spectateur, de la même façon que la mise en chaîne du montage cinématographique serait bientôt appelée à le faire. L'emploi du conférencier était par ailleurs la norme dans le panorama défilant et, de façon moins généralisée, dans le panorama circulaire. Les plans et les conférenciers faisaient ainsi office de guides d'orientation et fonctionnaient sur un mode s'apparentant à celui de la suite de tableaux et d'intertitres dans les reconstitutions cinématographiques des premiers temps.

La promotion entourant le panorama circulaire et le panorama défilant insistait par ailleurs sur la notion même de reconstitution. En misant sur l'aspect «reconstitution" du dispositif, celui-ci n'apparaissait plus seulement comme procédé capable d'encadrer la vision humaine, mais comme technologie en mesure de reproduire la réalité de façon proprement mimétique. On supposait aussi que le public n'apprécierait pleinement l'illusion produite par le panorama que si les sujets illustrés revêtaient un caractère foncièrement monumental et grandiose. En d'autres mots, les sites et les événements peints par les "panoramistes" devaient se montrer dignes de ce mode épique de représentation: "De grands sujets pour de grandes peintures ${ }^{6}$." 


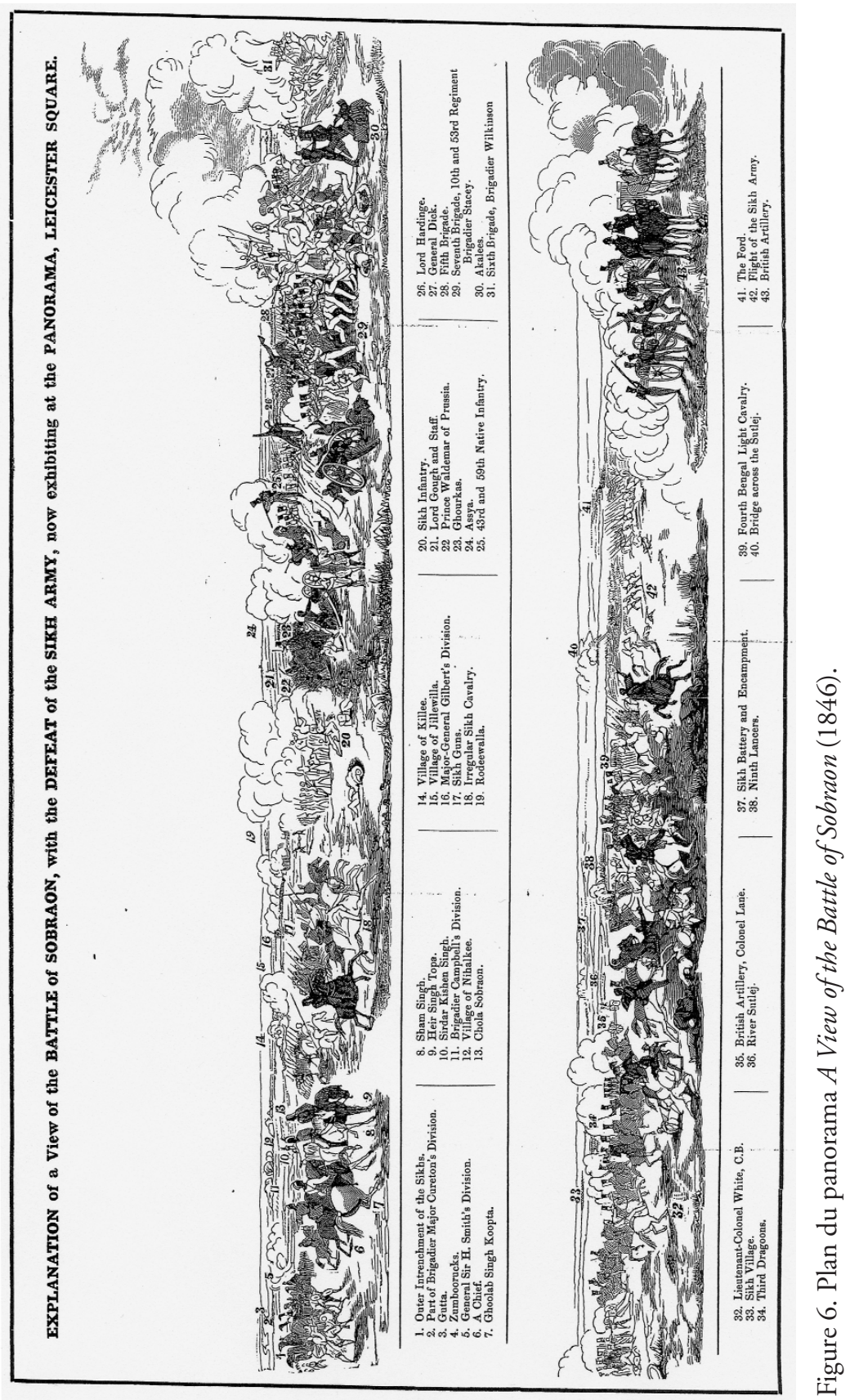


Les panoramas de batailles du XIX ${ }^{\mathrm{e}}$ siècle, ou la révision du passé

La représentation des grandes batailles était un genre particulièrement prisé des "panoramistes" (que ce soit pour le panorama circulaire ou le panorama défilant). Cette prédilection marquée pour la guerre semble paradoxale puisque, parmi tous les sujets possibles, les batailles comptaient assurément parmi ceux qui, vu l'abondance des actions qu'elles supposent, se prêtaient le plus difficilement à pareil type d'illustration. Il existait, au XIX siècle, deux types de panoramas de batailles: le panorama de type "journal illustré» et le panorama de type "monument national». Le premier représentait en images les nouvelles marquantes du jour et il était généralement exposé dans des rotondes construites à cet effet dans les capitales européennes. Le second était élaboré, au plan du discours, comme une peinture commémorative susceptible de célébrer une victoire pouvant remonter à plusieurs années (figure 7).

Les panoramas de type journal illustré apparaissaient souvent quelques semaines à peine après que la nouvelle d'une bataille importante eut atteint les rives britanniques (même si la bataille elle-même avait pu se dérouler plusieurs mois avant que la nouvelle ne parvienne jusqu'à Londres). Voulant exploiter l'enthousiasme manifesté par la population envers l'une de ces victoires, le "panoramiste» anglais Robert Ker Porter présenta en 1801 The Storming of Seringapatam, une peinture faisant 270 degrés - six semaines à peine après que la nouvelle concernant l'événement fut parvenue en Grande-Bretagne. Même si la nouvelle de la victoire britannique avait mis plusieurs mois à se propager du sud de l'Inde jusqu'à l'Angleterre, Porter réagit rapidement en créant un panorama dont l'impact se rapproche d'une certaine manière de celui d'un bulletin d'information $\mathrm{CNN}$, en raison notamment de sa capacité à assurer, grâce au réalisme accentué de la représentation, le transport virtuel du spectateur sur la ligne de front ${ }^{7}$.

$\mathrm{Au} \mathrm{XIX}^{\mathrm{e}}$ siècle, le spectateur qui visitait un panorama typique dépeignant une bataille n'était pas uniquement diverti par le côté spectaculaire de la peinture; il se transformait selon toute évidence en témoin historique ou en journaliste de guerre. En 


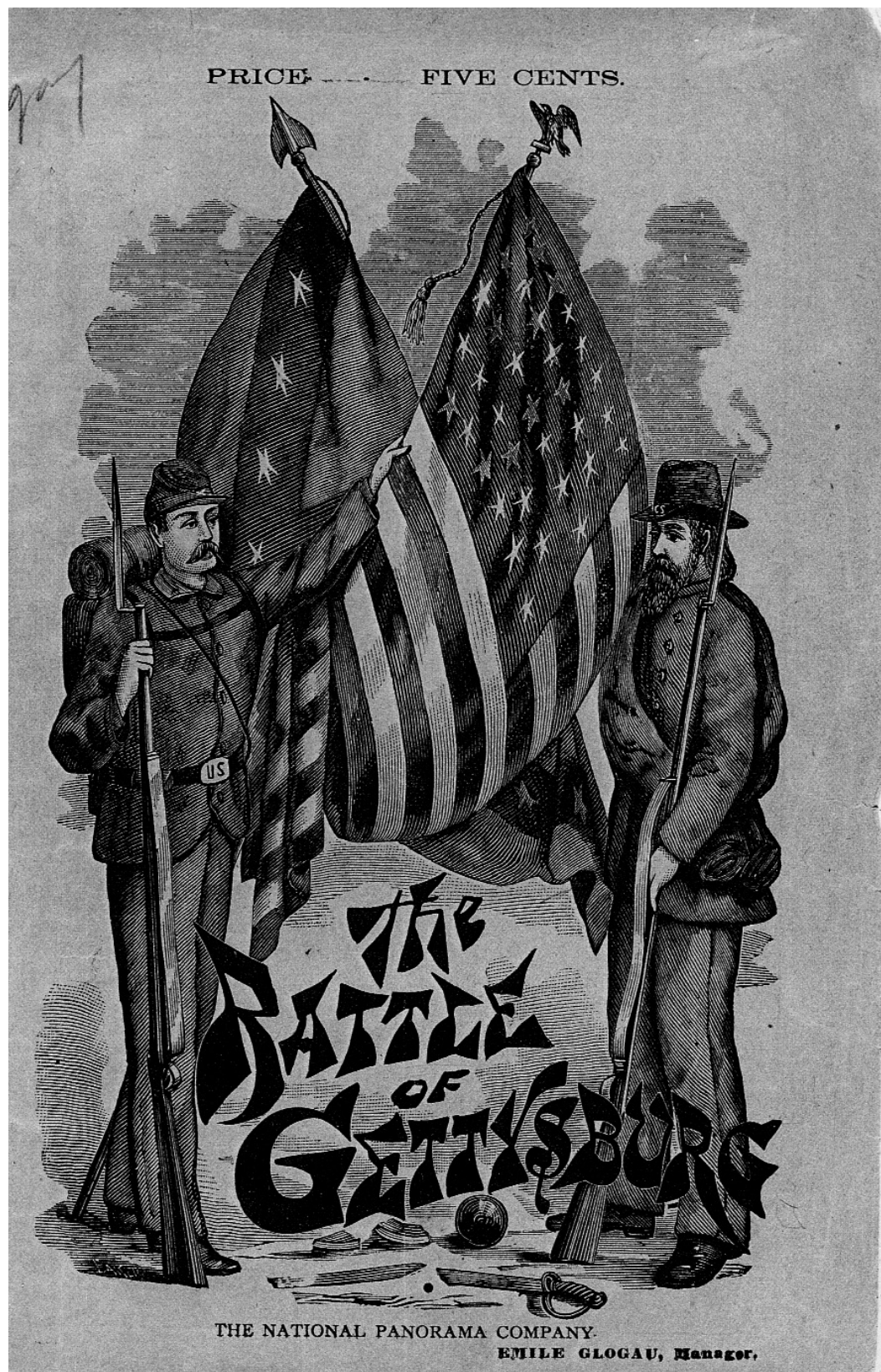

Figure 7. Couverture intérieure du guide accompagnant le Panorama of the Battle of Gettysburg. Chicago, The National Panorama Company (vers 1883). (C) Minnesota Historical Society 
fait, cette possibilité de revivre un événement d'une grande importance nationale, de mettre un pied dans l'histoire - position métaphoriquement représentée par la situation des spectateurs et par leurs déplacements physiques sur la plate-forme centrale d'observation - était sans doute pensée de façon à déclencher une poussée de ferveur nationaliste chez les spectateurs du début du XIX siècle. On ne doit donc pas sousestimer la fonction propagandiste des panoramas de Porter: installés en plein centre commercial et politique de la première puissance colonisatrice, ces panoramas illustrant les victoires militaires et la suprématie coloniale visaient, par leur transformation du fait guerrier en spectacle visuel, à renforcer l'opinion publique vis-à-vis de l'Empire. Lord Nelson a d'ailleurs reconnu sa dette envers le panoramiste Robert Barker, déclarant que l'œuvre de ce dernier avait "prolongé de toute une année la notoriété de sa victoire dans la bataille du Nil» (figure 8). L'interprétation de la bataille de Waterloo par Barker fut quant à elle couronnée d'un tel succès qu'il put quitter son métier de peintre de panoramas et vivre des profits générés par cette œuvre $^{8}$. Les quelques rares panoramas en opération de nos jours perpétuent ce rôle idéologique: comme le constate l'historienne hollandaise Evelyn Onnes-Fruitema, huit des dix panoramas construits depuis 1960 représentent des batailles nationales?

La célèbre peinture de la rivière Mississippi exécutée par John Banvard constitue un autre exemple de l'utilisation du panorama comme journal illustré. Comme John Hanner l'a fait remarquer, la guerre de Sécession américaine avait entraîné un renouvellement d'intérêt à l'égard du panorama de Banvard, ce qui avait en retour incité ce dernier à substituer aux sections consacrées à l'Ohio et au Missouri une nouvelle section intitulée "nouvelles opérations navales et militaires [sur le Mississippi]» (Banvard 1862). Que Banvard ait eu si peu de scrupules à insérer des événements tirés de l'actualité dans la composition du panorama illustre à la fois le besoin pour l'exploitant d'attirer de nouveaux spectateurs (et de faire revenir les habitués) et le statut intermédiaire du panorama, entre art et journal illustré. Qui plus est, cette réorganisation des diverses sections du panorama vient anticiper le réagencement par les premiers exploitants 
Le panorama et les origines de la reconstitution cinématographique

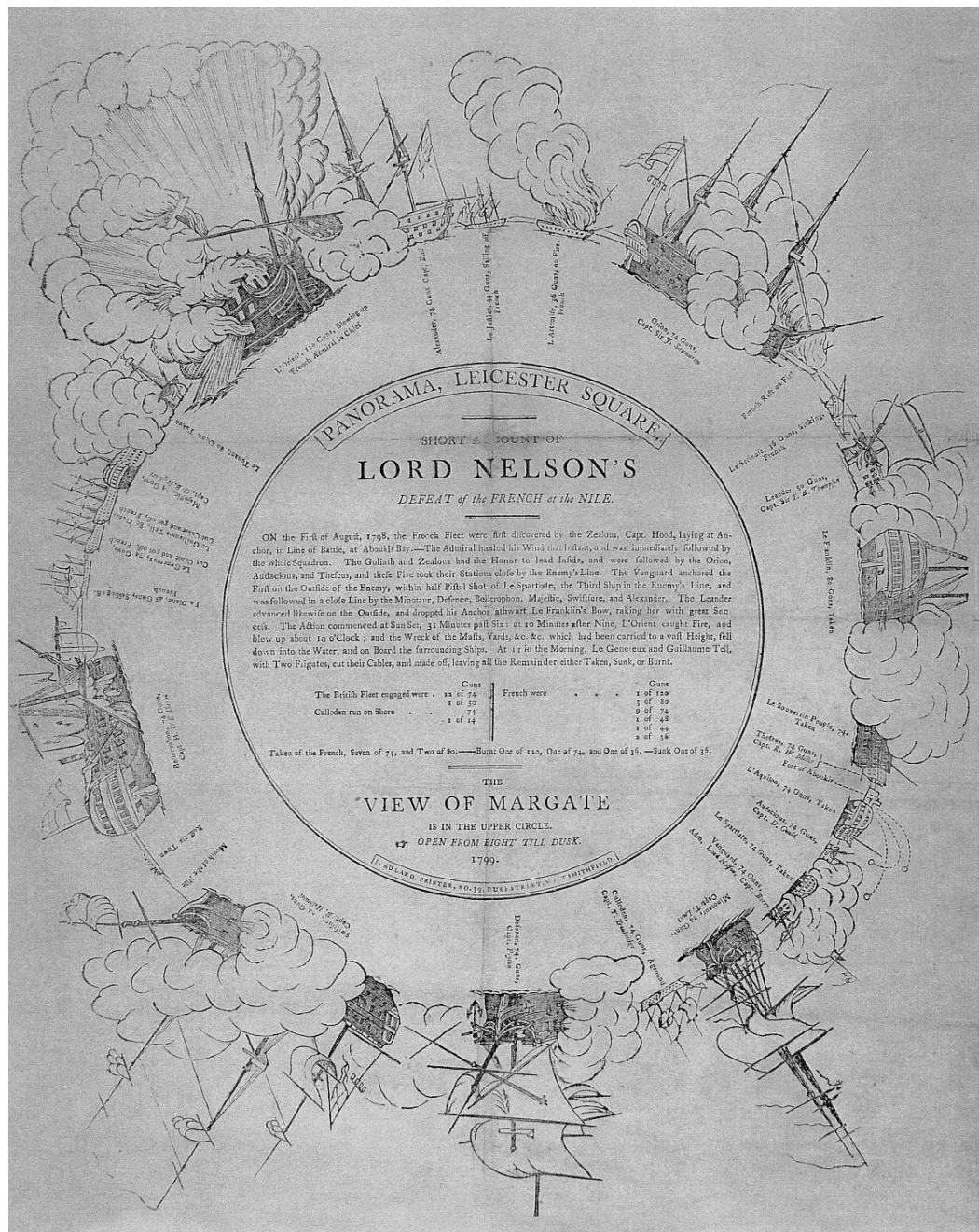

Figure 8. Explications du panorama The Battle of the Nile, montrant la victoire de Lord Nelson sur les Français. Le panorama A View of Margate était exhibé simultanément au niveau supérieur.

(C) Guildhall Library Corporation of London 
cinématographiques des bandes inscrites à leur programme, pratique qui leur permettait, selon les cas, d'élaborer une narration ou encore d'adapter leurs programmes en fonction de l'actualité et des intérêts du public.

Cependant, la représentation d'événements tirés des grands titres de l'actualité et l'insertion de prises de vues locales par les projectionnistes ambulants ne constituent pas les seuls aspects du cinéma des premiers temps qui viendront faire écho au panorama. En préfigurant les découpages propres aux premiers travelogues et en invitant à voir la peinture comme une représentation synthétique - néanmoins cohérente — d'un lieu et des gens qui le peuplent, le panorama en venait essentiellement à construire l'expérience du spectateur en termes protocinématographiques. Le matériel imprimé accompagnant View of the City of Cabul, Capital of Afghanistan (figure 9), panorama présenté en 1842 par Buford, identifie ainsi soixante-seize centres d'intérêt. Le plan d'orientation fourni au spectateur dirige dans un premier temps son regard vers une vue éloignée des montagnes du Kaffiristan, du Nejhau et du Taghau, pour ensuite le diriger vers des vues plus rapprochées de la population indigène (figures 46 à $76 \mathrm{du}$ plan). En plus des informations relatives aux heures d'ouverture et aux frais d'admission, le livret comportait de longues descriptions pour chacune des images numérotées sur la carte.

La stratégie consistant à combiner vues topographiques générales et représentations de personnages notables est reprise de manière frappante par le panorama View of the Battle of Sobraon, with the Defeat of the Sikh Army of the Punjab, également peint par Burford et installé au panorama de Leicester Square (figure 6). Sur le plan d'orientation qui accompagnait ce panorama, la peinture est répartie en deux sections, représentées l'une au-dessus de l'autre (même si le panorama devait être perçu comme une seule image continue). On peut apercevoir, dans chacune des deux sections du plan, les villages sikhs dans le lointain, ainsi que les troupes et l'armement des Britanniques et des Sikhs. On remarque par ailleurs que la page qui suit immédiatement le plan, dans la brochure, présente une vue plongeante sur l'artillerie des deux adversaires. Les 
Le panorama et les origines de la reconstitution cinématographique

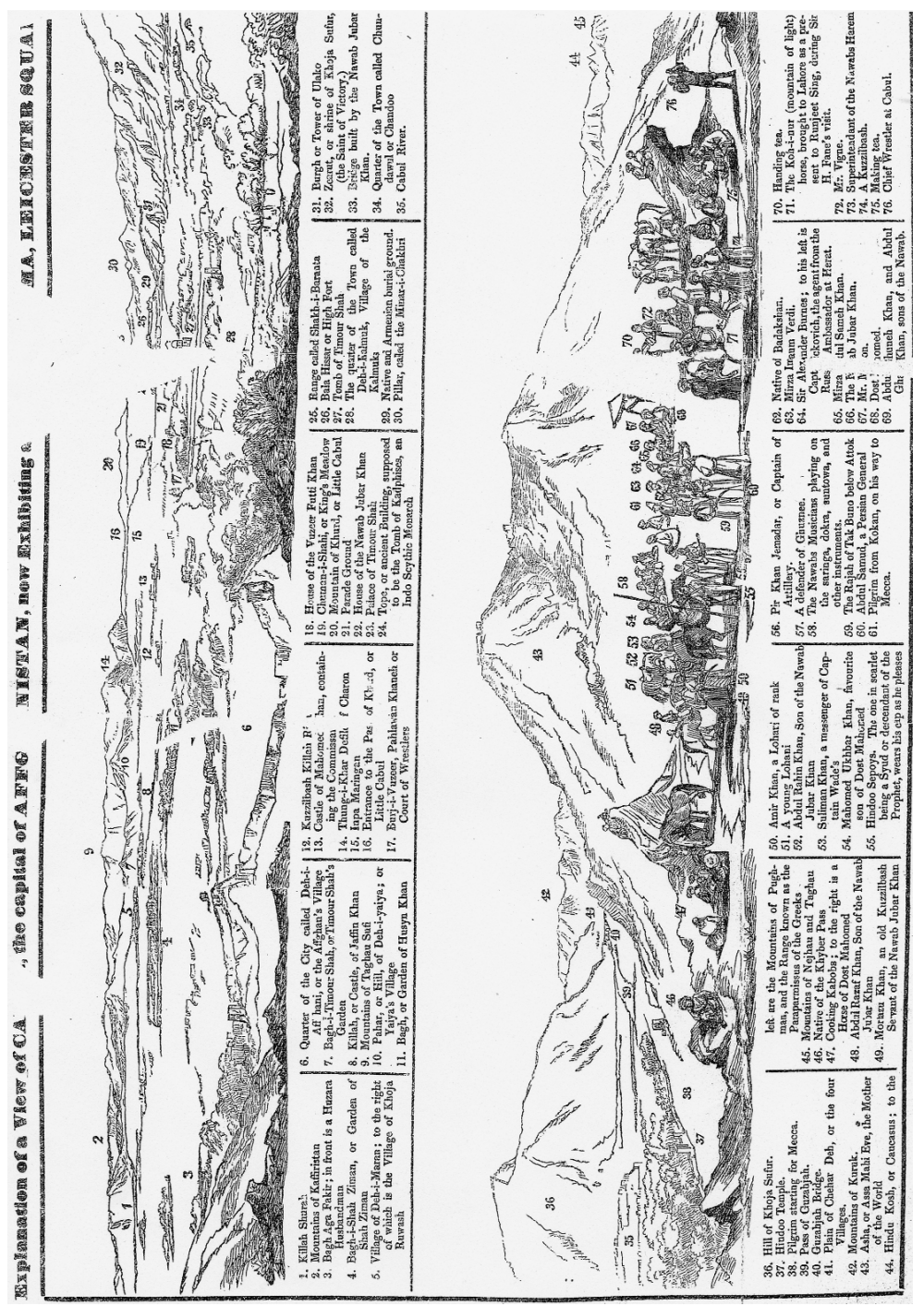

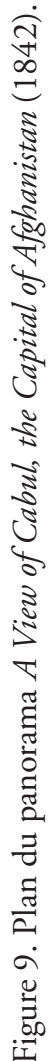


événements qui se déploient dans cette peinture semblent donc représenter le déroulement entier de la bataille. De plus, les nombreux détails peints en arrière-plan — trop petits pour être discernables dans le plan lui-même - donnent l'impression que l'action se déroule aussi bien sur un plan horizontal que latéral. Par son identification des personnages importants des deux camps et par l'amalgame qu'il offre des divers épisodes du conflit impliquant les deux armées, le panorama livre une représentation narrative et franchement complexe - pour ne pas dire chaotique - de la bataille. Bien qu'une multitude d'événements soient numérotés et décrits dans la brochure accompagnant le panorama, leur ordonnance ne semble être régie que par la seule organisation spatiale des éléments représentés sur la surface de la toile. Pour ceux des spectateurs qui se seraient procuré leur plan d'orientation avant leur visite du panorama (ou même pour ceux qui ne le consulteraient qu'après), l'identification des quarante-trois éléments numérotés pourrait s'avérer une tâche assez lourde. Au fond, la brochure permettait au visiteur de s'orienter, au cours de sa visite, afin de faciliter son observation du panorama. Elle pouvait aussi agir comme souvenir de la visite, lors d'une consultation a posteriori.

L'agencement et l'identification des lieux et des personnages représentés par le panorama de Sobraon semblent indiquer que les panoramas de ce type étaient conçus pour être examinés dans une séquence prédéterminée - un fait d'une importance cruciale dans le cadre de notre réflexion sur les propriétés protocinématographiques du panorama. Le plan d'orientation du panorama de Robert Budford, intitule Description of a View of the City of Nanking, and the Surrounding Country, présenté au panorama de Leicester Square en 1845, sert autant à situer le spectateur dans un espace général quà guider son regard de la gauche vers la droite (dans le sens des aiguilles d'une montre), d'un bout à l'autre de la peinture. Il faut bien sûr prendre soin de ne pas pousser l'analogie trop loin, mais il nous semble que la mise en séquences proposée dans le plan d'orientation partage certains traits essentiels avec celle qui sera bientôt exploitée par les premiers travelogues. La vue animée s'ouvrait généralement sur un plan général, qui permettait de situer le paysage, et l'on 
enchaînait ensuite sur une série de plans plus rapprochés montrant de manière synecdochique divers éléments architecturaux et certains spécimens de la population autochtone.

La peinture de Burford introduit d'abord le paysage et les édifices, puis l'artiste enchaîne avec les représentations de plusieurs dignitaires, tels le Chef ( $\mathrm{n}^{\circ} 29$ sur le plan d'orientation), les Parieurs chinois ( $n^{\circ} 30$ ), Nieu Kien, gouverneur de Nanking ( $n^{\circ} 33$ ), et ainsi de suite jusqu’à l'événement principal, une rencontre entre "Sa Majesté britannique", les commandants en chef de l'armée et les trois commissaires impériaux chinois. Alors qu'une représentation cinématographique des mêmes événements aurait été ponctuée de raccords venant marquer les multiples ellipses temporelles et spatiales, le panorama parvient à proposer un discours du même ordre par des procédés narratifs qui lui sont propres et que les spectateurs de l'époque étaient sûrement en mesure de décoder. En d'autres mots, le spectateur du panorama comprenait certainement que la peinture constituait une vue composite de Nanking plutôt qu'une vue à angle de 360 degrés d'un événement se déroulant dans un espace-temps unifié. Et pour les spectateurs proches de la confusion, l'avertissement imprimé dans le livret ne laissait plus de place au doute: "aucune rencontre du genre [n'a pris] place exactement à l'endroit représenté ici.» Burford défendait sa stratégie composite en invoquant "l'opportunité [offerte par cette technique] de présenter en un seul tableau tout aussi bien des portraits des principaux personnages impliqués dans les négociations qu'un reflet, plein de vitalité mais fidèle, des costumes et des mœurs de ce peuple singulier». Pour réaliser un portrait à la fois vivant et d'actualité, Burford amène le spectateur à partager avec lui une vue rapprochée de la vie de Nanking, en identifiant en cours de route les personnages clés, avec un souci semblable à celui du journaliste.

Le mélange de topographique et d'anecdotique proposé par le panorama menaçait toutefois de venir miner la présomption de vraisemblance sur laquelle reposait son fonctionnement. Même si Burford demeurait conscient du fait que la réception de son panorama se trouvait largement structurée par une certaine conception du réalisme historique, il vit dans la vue composite 
la possibilité d'un plus grand engagement et d'un plus grand intérêt du public. Il s'attela donc, avec cela en tête, à la création d'une représentation de la vie de Nanking et de ses environs, dont les caractéristiques allaient, selon moi, préfigurer le mode d'adresse cinématographique. Les diverses innovations apportées au panorama au cours de son évolution préparèrent de cette façon les spectateurs à composer avec une vision plus fragmentée du monde, une perception moderne informée tant par l'influence des journaux illustrés que par le désir du public de voir les lieux et les personnages importants qui ont marqué l'histoire. L'historien du théâtre Martin Meisel (1983, p. 61) soutient d'ailleurs que, "en dépit de la présomption de synchronie, le facteur "temps" pouvait s'infiltrer dans la scène représentée, par le truchement d'une incorporation discrète de certaines phases de l'action». En décidant de construire une vue composite plutôt qu'une vue à angle de 360 degrés conforme à la réalité géographique, Burford rejetait implicitement le principe fondateur du panorama, celui de la vue circulaire d'un lieu unique. Cependant, comme le public semblait apprécier toujours davantage l'"expérience" du panorama, même si la scène présentée ne s'accordait pas à la réalité continue d'un point d'observation unique, Burford dut juger cette dérogation amplement motivée.

Le panorama Description of a View of Baden Baden (1843), toujours de Burford, offre un autre exemple où des considérations d'ordres narratif et humain opèrent une déformation de la logique spatiale. La seconde moitié de ce panorama est en effet consacrée à une seule scène, une vue de la "Hutte des Soupirs", où se déroule une cérémonie riche en détails ethnographiques. En consacrant près de la moitié de la peinture à la représentation d'un seul événement, ce panorama anticipe à n'en point douter l'organisation générale de plusieurs films ethnographiques des premiers temps, dans lesquels les images initiales présentaient la topographie environnante, pour ensuite céder le pas à des plans plus détaillés sur les indigènes. En se dérobant à un type de représentation se voulant le reflet d'un seul lieu et d'un seul moment, en rejetant la contrainte du mimétisme lié à l'adoption d'un point de vue unifié, le 
panorama en question s'approche encore davantage du cinématographique. Bien entendu, la date associée aux derniers panoramas examinés (la décennie 1840) témoigne d'une certaine évolution du média. Confrontés à la compétition des panoramas défilants et des dioramas, les peintres de panoramas circulaires durent se sentir contraints d'ajouter plus d'éléments narratifs à leurs œuvres, d'accroître le dynamisme de celles-ci. Familier de ces attractions depuis plus d'un demi-siècle, le spectateur du panorama en était sûrement venu à espérer plus qu'une simple vue hyperréaliste - situation reflétant une fois de plus le passage des premières vues animées statiques aux premiers films pluriponctuels.

\section{Mort et illusion : quelques réflexions finales sur le panorama considéré comme trompe-l'œil}

De par son statut liminal, oscillant entre fiction et réalité, entre absence et présence, la reconstitution semble partager certaines propriétés avec le moment de la mort (et évoque ainsi le " "complexe" de la momie" qui se cache, selon Bazin (1958, p. 12), derrière l'invention du cinéma), c'est-à-dire ce moment où le corps, s'il continue toujours à se manifester dans le monde, ne peut plus être considéré comme lui appartenant pleinement ${ }^{10}$. À cet égard, la reconstitution partage de toute évidence un grand nombre de caractéristiques avec les expositions des musées de cire. En effet, non seulement la reconstitution constitua-t-elle tout au long de la seconde moitié du XIX ${ }^{e}$ siècle un principe organisateur très répandu dans les attractions offertes par les musées de cire, mais les personnages de cire étaient eux-mêmes plus que susceptibles, de par leur aspect lustré et humide rappelant la pâleur cadavérique, d'évoquer au plan phénoménologique l'idée de la mort suspendue ou trompée. Ainsi, bien que les figures de cire et les panoramas aient cherché à supprimer le moment de la mort, ils ne furent jamais en mesure d'échapper à cette sinistre référence.

Popularisées par Madame Tussaud, parallèlement à l'émergence du panorama, les musées de cire puisaient dans les nouvelles du jour (surtout celles traitant de la monarchie et d'exploits militaires) afin d'élaborer des vignettes narratives pouvant 
être, précisément, reproduites en cire. La même rhétorique pédagogique utilisée pour vanter les vertus éducatives et morales des panoramas fut employée dans la publicité des musées de cire ${ }^{11}$. Partie intégrante de ce processus de "spectacularisation de la réalité " décrit par Vanessa Schwartz, les figures de cire se prêtaient particulièrement bien à l'intertextualité. Le musée Grévin présentait ainsi à Paris un tableau constitué de figures de cire intitulé "Les coulisses d'un panorama ", qui montrait le peintre militaire Édouard Détaille appliquant les dernières touches à son panorama de la bataille de Rezonville, et cela même si le musée devait se retrouver en concurrence directe avec les panoramas ${ }^{12}$.

Plusieurs commentateurs ont évoqué dès cette époque le thème de la mort en tentant d'éclaircir la nature ambiguë de la reconstitution, dans les figures de cire comme dans les panoramas. La mort apparaît d'emblée comme une métaphore tout à fait propice lorsque sont abordés les panoramas ou les reconstitutions, compte tenu de cette obsession marquée pour les grandes batailles du XIX siècle dans les panoramas fixes ou défilants (figure 10). Aujourd'hui encore, dans un monde marqué par la télévision satellite et les chaînes en diffusion continue, la reconstitution et le thème de la mort sont fréquemment mis en rapport l'un avec l'autre dans des émissions relevant de l'infospectacle. La reconstitution s'avère en effet l'une des principales stratégies utilisées par les «true-crime programs", ces émissions fort répandues dans lesquelles des acteurs recréent des meurtres ou autres méfaits, espérant ainsi stimuler la mémoire du public et générer de nouvelles pistes d'enquête ${ }^{13}$.

Au-delà de sa représentation littérale dans plusieurs panoramas, le spectre de la mort semble faire partie du mode même de représentation de ce média: s'il revêt en surface l'apparence d'un être vivant, le cadavre, tout comme la toile hyperréaliste, n'en demeure pas moins dépourvu de vie. La reconstitution fait, au fond, état d'un paradoxe: d'une part, elle partage une étrange ressemblance avec la mort et, d'autre part, elle semble dotée du pouvoir de ramener la vie. À titre de «moment que nul ne peut décrire, d'événement auquel nul ne peut échapper, de processus 


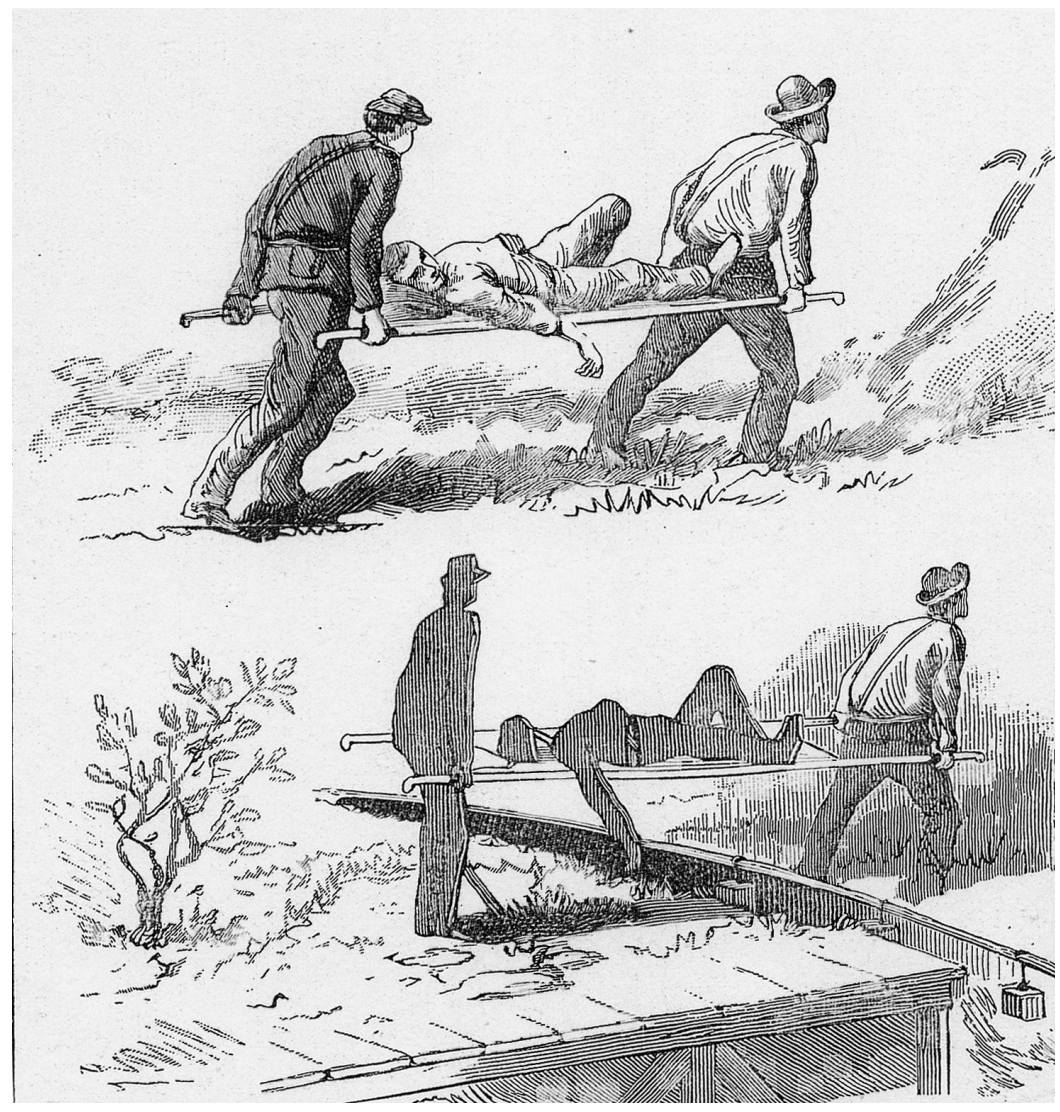

Figure 10. Utilisation de figures et d'accessoires de bois dans le panorama de Gettysburg, qui, en se fondant dans la scène peinte sur la toile, créent un effet tridimensionnel. 
que nul ne peut raconter" - pour reprendre les mots de Mieke Bal (1991, p. 362) — la mort se tourne elle aussi vers la reconstitution afin de se départir de son caractère définitif, la reconstitution étant en mesure de montrer tant ce qui échappe à la représentation que ce qui ne saurait être capté sur le vif. Le panorama peut de cette façon incarner la mort tout en la rejetant, puisqu'il se consacre à la construction d'un univers figé, agissant comme fac-similé d'un lieu ou d'un événement réel. De la même façon que la mort se trouve à poser un défi à la représentation, oscillant selon Bal (1991, p. 375) "entre un état et un événement", le panorama flotte entre différents états : c'est à la fois une sensation, une expérience et une représentation bidimensionnelle.

Si les rapports conflictuels qu'entretient la reconstitution panoramique avec le réel et l'imaginaire sont tributaires de cette impression macabre, le champ visuel offert par le panorama circulaire, avec son mouvement pétrifié, son échelle gigantesque et son effet d'immersion, était également en mesure d'exacerber l'aura de mort projetée par le dispositif. Celui-ci pouvait ainsi faire vivre aux spectateurs une expérience d'un caractère tout aussi sublime que stupéfiant, que certains n'hésitèrent pas à comparer à une expérience extracorporelle entraînant le spectateur aux frontières de la mort. Au moins un spectateur d'un panorama circulaire en vint à suggérer que l'on pouvait éprouver un "sentiment se rapprochant de celui de la mort" lors de la contemplation de la toile, et cela plus particulièrement lorsque la peinture incorporait de nombreuses scènes d'action. Admettant que la même chose pourrait, dans une certaine mesure, être vraie des peintures non panoramiques, l'auteur de ce commentaire ajoutait que les panoramas exigeaient beaucoup plus de leurs spectateurs que les tableaux des galeries:

On peut voir l'eau jaillir, tourbillonner à ses pieds, mais nul son ne se fait entendre, aucun écho du vacarme et du fracas accompagnant une grande cascade... On peut observer un groupe de touristes goûtant la vue des eaux tumultueuses, mais tout demeure immobile; on se retourne pour les retrouver figés comme dans la mort (Telbin 1900, p. 557 — c'est l'auteur qui souligne; notre traduction). 
De la même façon que Maxime Gorki prête au théâtre d'ombres des premières vues animées une qualité fantomatique et macabre, certains spectateurs du panorama rapportèrent que l'inquiétante immobilité des toiles, leur échelle et leur réalisme spectaculaires ajoutaient une sorte de solennité funéraire à la représentation ${ }^{14}$. À l'instar des dioramas utilisés dans les musées d'histoire naturelle pour représenter divers habitats, les panoramas étaient dotés d'un statisme particulier qui plaçait le spectateur au centre d'une scène "embaumée " optiquement. L'effet produit s'avérait désorientant, parfois même carrément troublant puisque, à la différence des surfaces planes du panorama défilant ou de l'écran du cinéma, sur lesquelles le spectateur ne fait que poser le regard, le panorama circulaire plaçait son spectateur au centre d'un univers reconstruit, lui faisait respirer un air fantomatique qu'aucun son ne venait troubler, si ce n'est les murmures étouffés des autres spectateurs. Cependant, même si l'absence de son diégétique contribuait sans doute à l'impression funèbre produite par les panoramas (et par les toutes premières vues animées), tous les panoramas n'étaient pas présentés ainsi. En fait, comme ce fut également le cas avec les vues animées, les panoramas défilants étaient rarement présentés dans un environnement silencieux, tandis que les panoramas circulaires étaient quant à eux souvent accompagnés de musique. Il n'était pas exceptionnel, cependant, d'émerger du sombre corridor menant au belvédère d'observation dans un silence funèbre, comme en fait foi le témoignage d'époque rapporté plus haut ${ }^{15}$. Dans sa description d'une expérience spectatorielle datant de 1900 - longtemps après les beaux jours du panorama W. Telbin rapporte que «les spectateurs, en sympathie avec ce monde immobile, s'y expriment à mi-voix; railleries futiles, commérages et autres bavardages ne se font plus entendre...". Peut-être davantage que les panoramas de batailles, les scènes illustrant les splendeurs du monde naturel semblent avoir suscité un regard contemplatif, que l'on pourrait associer à une cérémonie solennelle, comme des funérailles. Pour Bruno Ernst (1996, p. 123), l'expérience du panorama (en l'occurrence, du panorama Hendrik Mesdag, de La Haye) est "une expérience faite de calme et de paix ", un mémento évoquant pour les 
spectateurs du XIX ${ }^{e}$ siècle à la fois la nature transitoire de la vie et leur propre mortalité.

\section{Conclusion}

D'aucuns diront que le panorama, en tant que forme de divertissement populaire, s'est fait damer le pion par les vues animées à la fin du XIX siècle. Le panorama n'anticipe pas moins le cinéma à plusieurs égards. Il partage en effet avec les vues animées plusieurs caractéristiques phénoménologiques. Tout comme un film, un panorama est une représentation hyperréaliste, sur une toile grand format, qui n'est pas sans rappeler le cinérama, ces écrans rectangulaires (appelés, justement, écrans panoramiques) introduits dans les années 1950, et, de nos jours, le procédé Imax Solido qui enveloppe le spectateur de son image projetée sur un dôme de 360 degrés ${ }^{16}$. Un choix de sujets presque exclusivement inspirés non pas de thèmes littéraires ou mythologiques, mais d'événements et de lieux réels, conférait par ailleurs au panorama des effets à la fois cathartiques et thérapeutiques. Le panorama pouvait ainsi offrir un moment de paix et de tranquillité au cour des grandes métropoles frappées par l'industrialisation, et cela même si le spectacle qu'il présentait était bien souvent celui des horreurs de la guerre. Une visite au panorama représentait donc, dès la seconde moitié du XIX siècle, un moment de répit dans la course effrénée de la vie moderne. Tout comme les premières salles de cinéma, le site du panorama était en mesure d'offrir aux membres de la classe ouvrière un lieu où il était possible de recouvrer un certain contrôle sur une vie quotidienne de plus en plus réglée par la chaîne de montage et les horaires des transports en commun. En échange d'un modeste prix d'admission, le spectateur pouvait échapper au stress de la vie urbaine et exercer, du haut de la plate-forme d'observation centrale, un contrôle total sur la scène embrassée par son regard, se plaçant ainsi au centre d'un univers factice qui lui permettait de contempler le monde. D'ailleurs, cette impression de domination suscitée par un regard en plongée conférait probablement aux panoramas un caractère plus typiquement masculin, qui viendrait attester chez l'homme sa domination incontestée sur le monde. Mais il n'en demeure 
pas moins que l'espace du panorama était d'abord et avant tout un espace mixte: les femmes aussi, de toute évidence, appréciaient la prééminence visuelle offerte par la contemplation du panorama. En fait, on pourrait avancer que la présence de spectatrices sur les plates-formes des panoramas en vint à établir plusieurs pratiques spectatorielles toujours en vigueur à l'époque des premières vues animées.

Les panoramas préparèrent donc les spectateurs du XIX siècle à la réception d'images à grande échelle, ouvrant ainsi la voie au spectacle cinématographique. Bien évidemment, le panorama n'est pas le seul précurseur du cinéma digne de mention, aux plans esthétique et idéologique. Il a néanmoins légué un héritage qui continue de se faire sentir aujourd'hui, notamment dans cette quête d'illusionnisme absolu dans la représentation du monde, et plus particulièrement dans la représentation de sujets situés en dehors de l'expérience humaine ordinaire: ascension de l'Everest, voyage dans la navette spatiale Discovery, plongée sousmarine au large de l'archipel des Galápagos - pour ne citer que les plus récentes productions Imax. La fascination populaire pour les vues à angle de 360 degrés ne s'est jamais amoindrie; elle a simplement fluctué au cours des quelque deux siècles qui nous séparent du brevet obtenu par Robert Parker en 1787. Alors qu'Imax et les technologies Internet exploitant une vision circulaire consolident leurs positions au sein du paysage culturel et commercial contemporain, prenons soin de ne pas oublier leurs pittoresques ancêtres géants.

The City University of New York

Traduit de l'anglais par Louis Pelletier; texte français établi avec la collaboration de Nicolas Dulac

\section{NOTES}

1. Plusieurs sujets fictifs ont inspiré des panoramas. Parmi eux: Pilgrim's Progress, de John Bunyan et Paradise Lost, de Milton.

2. Pour plus d'information sur la genèse des panoramas et la notion de voyage virtuel, voir Griffiths 2004.

3. Voir Parry 1971 (p. 53). 
4. Voir Onnes-Fruitema et Zoetmulder 1981 (p. 18).

5. Certains points pouvaient facilement se passer de commentaire. Néanmoins, il ne semble pas y avoir de règle particulière permettant de déterminer quels éléments méritaient ou non une explication.

6. "Big subjects for big pictures."

7. Selon Oettermann, le panorama The Pandemonium of Boulogne, de John Thomas Serres, en «fournissant une information visuelle de dernière heure sur la situation militaire actuelle", constitue l'exemple le plus convaincant d'attraction qui présageait, au XIX ${ }^{e}$ siècle, le journalisme télévisuel contemporain. Voir OnnesFruitema et Zoetmulder 1981 (p. 125).

8. Voir Anonyme 1847 (p. 46-47). Les guerres napoléoniennes ont été représentées avec succès sous forme de panoramas, entre autres avec The Battle of the Nile (1798) et The Battle of Trafalgar (1805). Voir Andes 1930 (p. 59). Napoléon était impressionné par le pouvoir du panorama en tant qu'outil de propagande même si, comme le fait remarquer Lee Parry, ses plans pour la construction de huit rotondes exposant les grandes batailles de la Révolution et de l'Empire ont été contrecarrés par les événements de 1812-15. Voir Parry 1971 (p. 54).

9. Voir Onnes-Fruitema 1996 (p. 31). Par exemple: «En Irak, le panorama moderne Bataille d'Al-Qadissiyah représente une bataille ayant eu lieu en l'an 637 A.D.; après avoir emprunté un escalier ou un ascenseur jusqu'au belvédère d'observation, le spectateur se retrouve encerclé par la mêlée. " De façon à accentuer tant le spectacle que l'illusion, des effets sonores évoquant la guerre sont continuellement audibles en arrière-plan.

10. Voir également dans le même recueil «Le mythe du cinéma total» (Bazin 1958, p. 21-26).

11. Des considérations d'espace nous obligent à limiter l'analyse des multiples correspondances qui unissent panorama, reconstitution et figure de cire, sur lesquelles bien des choses restent à dire. Pour une discussion plus approfondie sur la combinaison d'éléments réalistes et spectaculaires particulière aux présentations de figures de cire, et plus spécifiquement aux expositions du musée Grévin de Paris à la fin du XIX siècle, voir Schwartz 1998 (p. 89-148).

12. Voir Schwartz 1998 (p. 90 et 104).

13. En Grande-Bretagne, Crimewatch constitue un exemple canonique d'émission du genre. Aux États-Unis, certaines émissions d'info-spectacle telles que America's Most Wanted et Inside Edition font, dans un cadre sensationnaliste, un large usage de la reconstitution.

14. Maxime Gorki, "Le Royaume des ombres." Originellement publié dans le journal Nijegorodskilistok, édition du 4 juillet 1896, et signé I.M. Pacatus (pseudonyme de Gorki). Traduction du russe à l'anglais par Leda Swan, publiée dans Jay Leda, Kino, London, Allen and Unwin Ltd., 1960. Le commentaire de Gorki se trouve également reproduit dans Kevin MacDonald et Mark Cousins, Imagining Reality: The Faber Book of Documentary, London, Faber and Faber, 1996, p. 6-10. Traduction française publiée dans Vertigo, no 15, 1996, p. 10.

15. Le panorama Mesdag, à La Haye, diffuse un commentaire dans lequel tous les points saillants de la peinture sont approfondis. Lors de l'achat du billet, les groupes de touristes qui s'apprêtent à visiter le panorama choisissent, parmi les cinq choix offerts (néerlandais, allemand, anglais, français et espagnol), la langue dans laquelle ils préferent entendre le commentaire. Lorsque le musée est peu achalandé, un disque compact reproduisant des effets sonores (cris d'oiseaux, sons de l'océan, etc.) se substitue au commentaire. Marijnke de Jong, directrice du panorama de Mesdag, remarque que certains visiteurs se plaignent du commentaire enregistré, qu’ils jugent 
inopportun et gênant. Bien que soucieuse de répondre aux demandes des visiteurs, la direction du panorama a opté pour le maintien du commentaire, compte tenu du fait que la majorité des visiteurs semble l'apprécier. Entrevue de l'auteur avec de Jong, 14 juillet 2001, La Haye.

16. Pour une analyse approfondie des liens unissant les panoramas et les technologies à écrans larges du type IMAX, voir Griffiths 2004.

\section{RÉFÉRENCES BIBLIOGRAPHIQUES}

Andes 1930 : Herbert C. Andes, "The Leicester Square and Strand Panoramas", Notes and Queries, vol. 159, n ${ }^{\text {os }} 4-5,1930$.

Anonyme 1847: "The Panorama, with Memoirs of Its Inventor Robert Barker, and His Son, the Late Henry Astor Barker », The Art Journal, vol. 9, 1847.

Arrington 1959: Joseph Earl Arrington, «William Burr's Moving Panorama of the Great Lakes, The Niagara, St. Lawrence and Saguenay Rivers ", Ontario History, vol. 51, n³, 1959.

Bal 1991 : Mieke Bal, Reading Rembrandt: Beyond the Word-Image Opposition, New York, Cambridge University Press, 1991.

Banvard 1862: John Banvard, Descriptions of Banvard's Geographical Painting of the Mississippi River, Extensively Known as the "Three Mile Pictures," with New Additions of the Naval and Military Operations on that River, Exhibiting a View of the Country 1,500 Miles in Lenght, from the Mouth of the Missouri to the Balize, New York, L.H. Bigelow, 1862.

Bazin 1958: André Bazin, "Ontologie de l'image photographique», Qu'est-ce que le cinéma? Tome I. Ontologie et langage, Paris, Cerf, 1958.

Ceram 1965: C.W. Ceram, Archéologie du cinéma, Paris, Plon, 1965.

Ernst 1996 : Bruno Ernst, "Perspective and Illusion", dans Yvonne van Eekelen (dir.), The Magical Panorama, Zwolle, Waanders Publishers, 1996.

Griffiths 2004: Alison Griffiths, "The Largest Picture Ever Executed by Man:" Panoramas and the Emergence of Large-Screen and 360 Degree Technologies ", dans John Fullerton (dir.), Screen Culture: History and Textuality, Eastleigh, John Libbey Press, 2004.

Heilbron 1936: Bertha L. Heilbron, "Making a Motion Picture in 1848: Henry Lewis on the Upper Mississippi ", Minnesota History, vol. 17, n² 1936.

Meisel 1983: Martin Meisel, Realizations: Narrative, Pictorial, and Theatrical Arts in Nineteenth-Century England, Princeton, Princeton University Press, 1983.

Onnes-Fruitema 1996: Evelyn J. Onnes-Fruitema, "Of Panoramas Old and New», dans Yvonne van Eekelen (dir.), The Magical Panorama, Zwolle, Waanders Publishers, 1996.

Onnes-Fruitema et Zoetmulder 1981 : Evelyn J. Onnes-Fruitema et Paul A. Zoetmulder, The Panorama Phenomenon, La Haye, Mesdag Panorama, 1981.

Parry 1971: Lee Parry, "Landscape Theater in America", Art in America, vol. 59, décembre 1971.

Schwartz 1998: Vanessa R. Schwartz, Spectacular Realities, Berkeley, University of California Press, 1998.

Squires 1933: Monas N. Squires, «Henry Lewis and His Mammoth Panorama of the Mississippi River", Missouri Historical Review, n 27, 1933.

Telbin 1900: W. Telbin, "The Painting of Panoramas", The Magazine of Art, vol. 24, 1900. 